\title{
Symbiotic stars on Asiago archive plates. II $^{\star}$
}

\author{
U. Munari ${ }^{1}$ and R. Jurdana-Šepić ${ }^{2}$ \\ 1 Osservatorio Astronomico di Padova, Sede di Asiago, 36012 Asiago (VI), Italy \\ 2 Physics Department, University of Rijeka, Omladinska 14, 51000 Rijeka, Croatia
}

Received 20 November 2001 / Accepted 23 January 2002

\begin{abstract}
The Asiago photographic archive has been searched for plates containing the symbiotic stars Hen 2-468, QW Sge, LT Del, V407 Cyg, K 3-9, V335 Vul, FG Ser and Draco C-1. A total of 635 plates imaging the program stars have been found and the brightness estimated using the Henden \& Munari $(2000) U B V(R I)_{\mathrm{C}}$ photometric sequences. These historical data have allowed for the first time the determination of the orbital periods of Hen 2468 (774 days) and QW Sge (390.5 days), a significant improvement in the orbital period of LT Del (465.6 days) and for V407 Cyg an evaluation of the Mira's pulsation period and complex lightcurve shape in the red $(R$ and $I$ bands). Some previously unknown outbursts have been discovered too.
\end{abstract}

Key words. stars: binaries: symbiotic

\section{Introduction}

In Paper I (Munari et al. 2001) we presented the first results of the search for plates in the Asiago photographic archive containing symbiotic stars. A total of 602 plates imaging AS 323, Ap 3-1, CM Aql, V1413 Aql, V443 Her, V627 Cas and V919 Sgr were located and the brightness of symbiotic stars estimated at the microscope against the $U B V(R I)_{\mathrm{C}}$ comparison sequences calibrated by Henden \& Munari (2000). The effort paid fruitful dividends on these poorly known systems: AS 323 was discovered to be quite probably an eclipsing system with the shortest known orbital period among symbiotic stars $(P=197.6$ days $)$, and CM Aql was found to have a $P=1058$ days orbital period and a remarkable amplitude of the associated reflection/heating effect $(\Delta B \sim 2.2 \mathrm{mag})$.

In this second paper of the series we searched the Asiago archive for plates imaging the eight symbiotic stars Hen 2-468, QW Sge, LT Del, V407 Cyg, K 3-9, V335 Vul, FG Ser and Draco C-1. A total of 635 plates were found containing the program stars and their brightness has been again estimated at the microscope against the $U B V(R I)_{\mathrm{C}}$ comparison sequences calibrated by Henden \& Munari (2000).

The time scale of variability for symbiotic stars is quite long: the orbital periods range from $\sim 1$ year up to several

Send offprint requests to: U. Munari, e-mail: munari@pd.astro.it

* Table 2 is only available in electronic form at the CDS via anonymous ftp to cdsarc.u-strasbg.fr (130.79.128.5) or via

http://cdsweb.u-strasbg.fr/cgi-bin/qcat?J/A+A/386/237 decades while rise and decay from an outburst may take anything from a few years to more than a century (cf. Kenyon 1986; Mikolajewska 1996). Such times scales are generally too long for devoted photometric programs to be carried out by a single Institute, but are instead well handled by those patrol programs that run for at least several decades at some Observatories around the world, one of them being Asiago. To stimulate and facilitate inspection of plates at the various archives in the world, Henden \& Munari $(2000,2001,2002)$ have so far calibrated accurate and deep $U B V(R I)_{\mathrm{C}}$ sequences around 60 symbiotic stars. The present series of papers applies these sequences to those plates in the Asiago archive found to contain symbiotic stars, with the aim of contributing our data to the effort of reconstructing the longest and most detailed possible historical lightcurves.

\section{Results}

\subsection{Hen $2-468$}

Discovered on objective-prism plates by Henize (1967) who noted a possible variability, its symbiotic nature was recognized by Carrasco et al. (1983) and Allen (1984) on the basis of optical spectra showing a late M-giant continuum and a rich emission line spectrum including HeII and the $6830 \AA$ symbiotic band. Allen (1974) determined its infrared brightness as $K=7.96, J-K=+1.48$. It was discovered to be a variable star by Margoni \& Stagni (1984, their variable \#3) that reported for the period 1969-1979 a brightness range $14.0 \leq V \leq 15.0$ and $16.0 \leq B \leq 17.2$. The star however has not yet received a variable star 

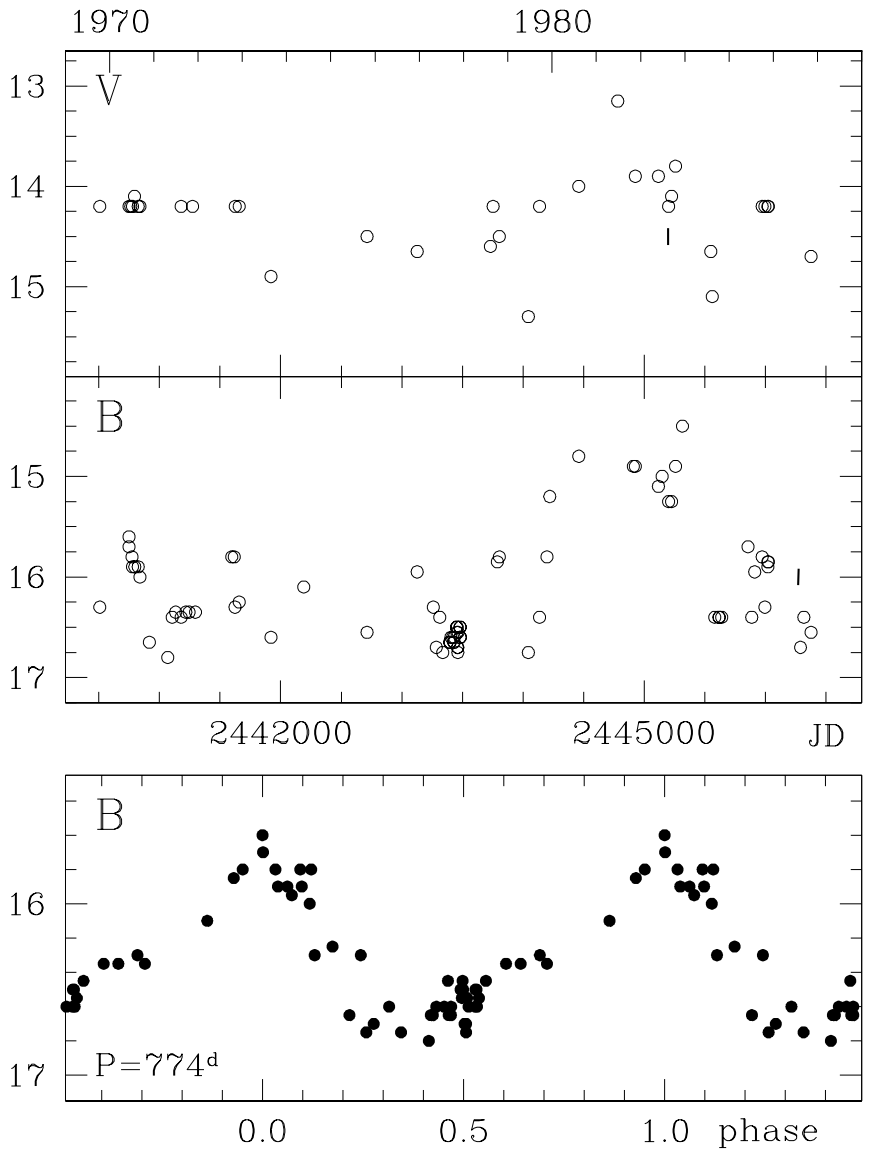

Fig. 1. $B$ and $V$ lightcurve for Hen 2-468. Vertical bars indicate "fainter than". Lower panel: phase plot of $B$ quiescence data (between 2440508 and 2444044) according to Eq. (1).

Table 1. The program symbiotic stars. Equinox J2000 and epoch $\sim 2000$ coordinates are from Henden \& Munari (2000).

\begin{tabular}{|c|c|c|}
\hline & $\alpha_{J 2000}$ & $\delta_{J 2000}$ \\
\hline Draco C-1 & 171957.661 & +575005.74 \\
\hline FG Ser & 181507.121 & -001851.96 \\
\hline K 3-9 & 184024.133 & -084357.73 \\
\hline V335 Vul & 192314.124 & +242740.17 \\
\hline QW Sge & 194549.548 & +183648.47 \\
\hline LT Del & 203557.234 & +201127.91 \\
\hline Hen $2-468$ & 204118.989 & +344452.52 \\
\hline V407 Cyg & 210209.831 & +454632.85 \\
\hline
\end{tabular}

name. Infrared photometry by Munari et al. (1992) gave $K=8.02, J-K=+1.50$, almost identical to Allen (1974), suggesting that the cool giant in Hen 2-468 is not intrinsically variable. Henden \& Munari (2000) observed Hen 2468 in quiescence at $B=16.6$ and $B-V=+1.8$ in 1999 , in agreement with the Margoni \& Stagni (1984) values.

The Asiago plates (78 $\mathrm{B}, 34 \mathrm{~V}$ and $5 \mathrm{I}$ band) cover the time span between Oct. 1969 and Dec. 1987. The resulting $B$ and $V$ lightcurves are plotted in Fig. 1. On them, Hen 2-468 appears to have been in quiescence up to Oct. 1979, when it entered an outburst phase that lasted until the summer of 1985 when the system returned to mean quiescence brightness. The outburst amplitude was limited, at maximum reaching $\Delta B \sim 1.8$ mag above mean quiescence level. Given the red color in quiescence ( $B-V=+1.8$, cf. Table 2$)$, the outburst is barely traceable in the $V$ lightcurve dominated by the stable emission of the $\mathrm{M}$ giant $(B-V \sim+1.0$ at outburst maximum). This is the first-ever recorded outburst of Hen 2-468.

The $B$ quiescence lightcurve of Hen 2-468 looks stable in mean brightness $(<B\rangle=16.3)$ but highly variable around it. A period search was performed and revealed a strong 774 day periodicity. The absence of a corresponding modulation in the $V$ data and the shape of the phased quiescence $B$ lightcurve (presented in the bottom panel of Fig. 1) both support the interpretation of the $\Delta B \sim$ $1.1 \mathrm{mag}$ variability as a reflection/heating effect, with

$\operatorname{Max}(B)=2440749+774 \times E$

giving the times of maxima, corresponding to the passage at inferior conjunction of the white dwarf companion to the $\mathrm{M}$ giant. The asymmetric placement of the minimum at $\phi \sim 0.35$ could argue in favor of a moderately eccentric orbit. The present one is the first ever determination of the orbital period for Hen 2-468.

\section{2. $Q W$ Sge}

QW Sge (= AS 360) is another symbiotic star that has received scanty attention in the literature. Munari \& Buson (1991) already reviewed the latter and discussed the system properties based on IUE, optical and IR observations.

QW Sge has an optical companion 3.5 arcsec to the north, that Munari and Buson classified as an F0 V star with $B=13.59$ and $B-V=+0.45$. Henden \& Munari (2000) found different values for it $(B=13.18$ and $B-V=+0.83)$, with a large scatter of 0.25 mag between three different measurements (compared to the stability at a few millimag for nearby stars of similar brightness). This clearly indicates that the optical companion is itself a variable star, and this complicates the interpretation of photometry made with moderate or short focus telescopes that are not able to separate QW Sge from the close optical companion (as it is the case for most of the photographic plates in the archives around the world).

The 464 blue plates from the archive of the Sternberg Astronomical Institute in Moscow examined by Kurochkin (1993) suffer from this blending. All his measurements refer to QW Sge and the companion star merged in a single stellar image. Kurochkin's lightcurve covers the period 1898-1990, during which two outbursts took place: one extending from July 1962 to March 1972 with $B=11.5$ at maximum, the other from May 1982 to September 1989 with a much more complex lightcurve and a peak brightness $B=12.0$. In between the combined brightness of QW Sge and its nearby companion remains in the range $12.9 \leq B \leq 13.4$

Our measurements in Table 2 pertain to quiescence conditions for QW Sge. The favorable focal lengths of the Asiago Schmidt telescopes allow a marginal separation of QW Sge from the companion star. The measurements in 

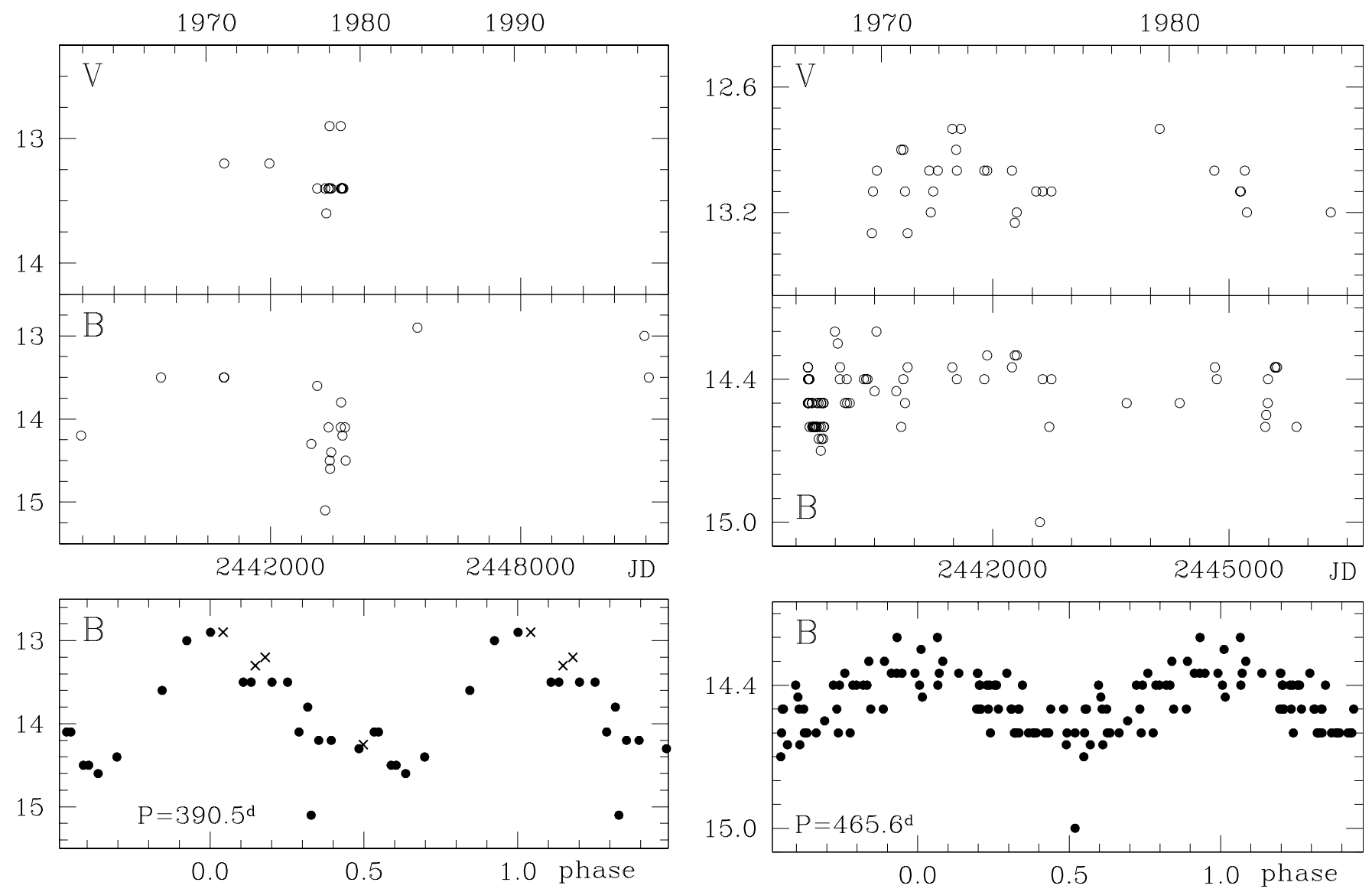

Fig. 2. $B$ and $V$ data for QW Sge. Lower panel: phase plot of $B$ data according to Eq. (2). Crosses indicate data from Henden \& Munari (2000), Munari \& Buson (1991), Palomar DSS-1 and DSS-2.

Table 2 pertain to QW Sge alone. A few more plates from the Asiago archive were not considered here because bad seeing conditions caused an unrecoverable merging of the two stellar images into a single one.

A period search was performed and a fine periodicity emerged following the ephemeris

$\operatorname{Max}(B)=2445528+390.5 \times E$

The Table 2 data are phase plotted in Fig. 2. Our measurements are limited in number and non-negligible errors are expected to affect our estimates of the nearly merged stellar pair. To test ephemeris (2) we therefore estimated QW Sge brightness on Palomar DSS-1 and DSS-2 blue plates ( $\mathrm{JD}=2433480$ and $B=13.3, \mathrm{JD}=2446326$ and $B=12.9$, respectively) and plotted them in Fig. 2 as crosses together with single $B$ measurements from Henden \& Munari (2000) and Munari \& Buson (1991). The agreement of these external data with those from the Asiago plates is excellent over the 5 decades spanned by the data. The lightcurve shape, its amplitude $(\Delta B=1.7 \mathrm{mag}$, and a few tenths in $V$ ) and the high repeatability over a long period of time suggests an interpretation in term of reflection/heating effect in phase with the orbital motion. The present $390 \mathrm{~d} 5$ day is the first ever determination of the orbital period of QW Sge.

Fig. 3. $B$ and $V$ lightcurve of LT Del. Lower panel: phase plot of $B$ data according to Eq. (3).

\section{3. $L T D e l$}

The history and photometric properties of LT Del up to the early 90ies have already been reviewed by Munari \& Buson (1992). The quiescent lightcurve of LT Del displays a strong reflection/heating effect with amplitudes $\Delta U=$ $+1.7, \Delta B=0.5$ and $\Delta V=0.2 \mathrm{mag}$, that Arkhipova \& Noskova (1988) reported to follow the ephemeris Min = $2445910+488 \times E$, later revised to $\mathrm{Min}=2445910+$ $478.2 \times E$ by Arkhipova et al. (1995). The latter listed $B=14.4$ and $B-V=+1.3$ as mean values for quiescence. Passuello et al. (1994) discovered the only outburst so far recorded for LT Del, with a $\Delta B \sim 1.6 \mathrm{mag}$ in 1994 .

Our lightcurve of LT Del extends from Sep. 1967 to Aug. 1985 (cf. Fig. 3), with mean values $B=14.41$ and $B-V=+1.32$ identical to those of Arkhipova et al. (1995) for LT Del in quiescence. A period search revealed a strong periodicity at $465^{\mathrm{d}} 6$, with an amplitude $\Delta B \sim 0.27 \mathrm{mag}$ and a sinusoidal shape indicative of a reflection/heating effect. The variation follows the ephemeris

$\operatorname{Max}(B)=2440493+465.6 \times E$

The $465^{\mathrm{d}} 6$ period differs significantly from previous estimates. The 488 days suggested by Arkhipova \& Noskova (1988) is definitively ruled out by our data, while the 478.2 days indicated by Arkhipova et al. (1995) performs 


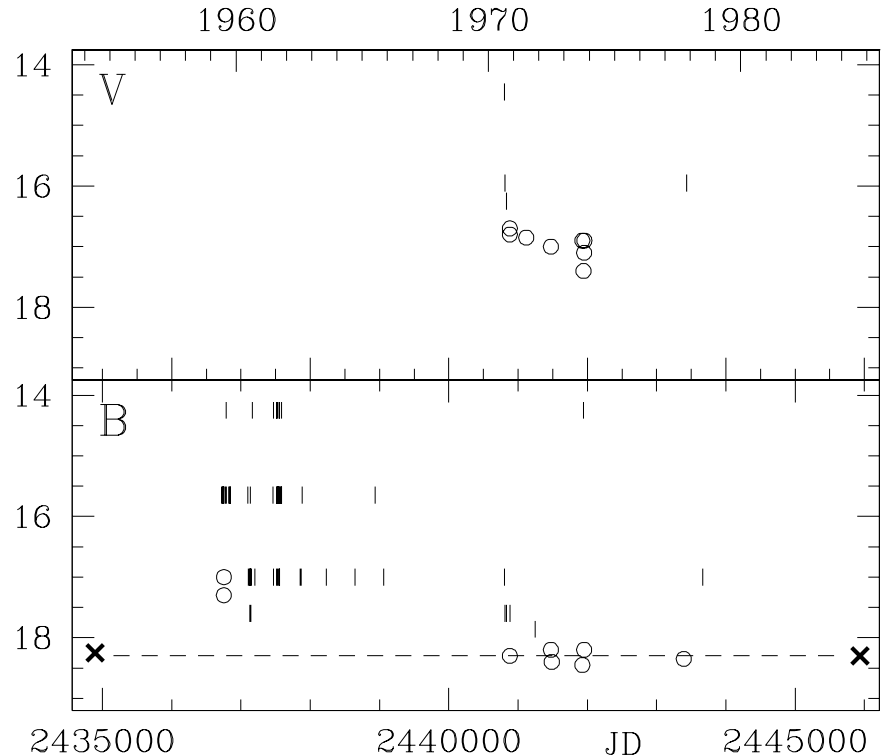

Fig. 4. $B$ and $V$ data of K 3-9. Vertical bars indicate fainter than. The two crosses indicate the brightness of $K 3-9$ on Palomar DSS-1 and DSS-2 blue plates, and the dashed line the 1999 quiescence $B=18.3$ level.

only marginally better with a high dispersion affecting the folded lightcurve.

\section{4. $K 3-9$}

The photometric properties, history and orbital period are unknown for this faint and poorly studied symbiotic star. Given its optical faintness, the scanty investigations were concentrated in the infrared and radio domains where the system is much easier to observe (Ivison \& Seaquist 1995). Henden \& Munari (2000) report an average $B=18.3$ and $B-V=+1.3$ for 1999 .

The lightcurve from Asiago archival plates presented in Table 2 and Fig. 4 covers the period from 1959 to 1979 and confirms the optical faintness and the moderately red color of K 3-9. The object has always been close to plate limiting magnitude, however a flat quiescence in the 70ies with $B=18.3$ and $B-V \sim 1.0$ and a brighter phase in 1959 at $B=17.2$ are well established. A $\sim 0.5$ mag variability affected K 3-9 in $V$ with apparently no counterpart in $B$.

Ivison \& Seaquist (1995) argued about the possible presence of a Mira in K 3-9 and an ongoing symbiotic nova outburst phase for the WD companion. Symbiotic Miras are normally discovered as such when the WD companion enters a powerful outburst that ionizes and lights-up by several magnitudes the circumstellar material producing a rich emission line forest and a flat blue continuum.

Symbiotic Miras with erupting WD companions show a slow, smooth and monotonic decrease in brightness following outburst maximum. For example, the extremely smooth decline of HM Sge and V1016 Cyg over the last 20 years has been characterized by $\Delta V \sim 0.05$ and

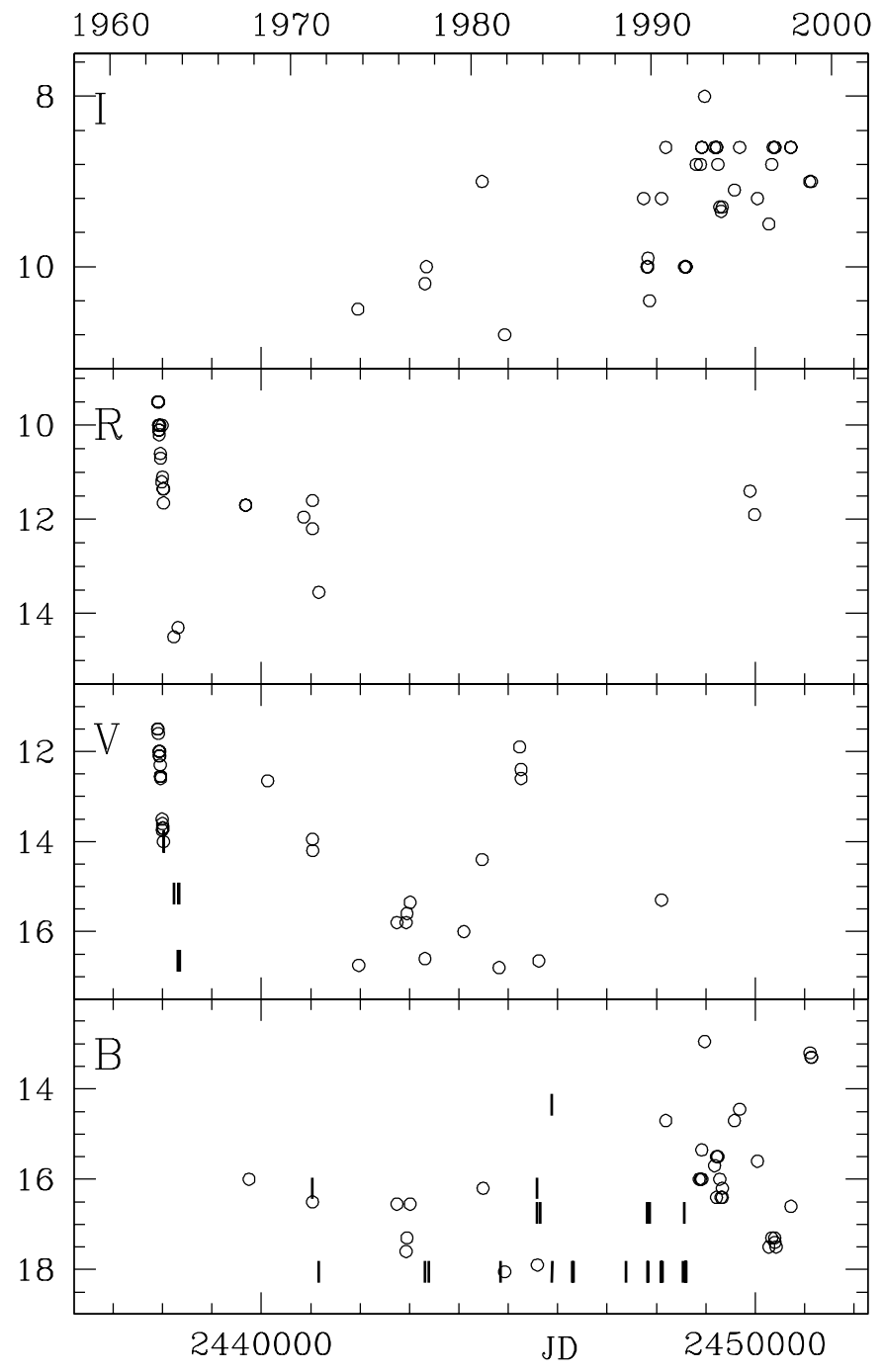

Fig. 5. $B, V, R$ and $I$ lightcurves of V407 Cyg. Vertical bars indicate fainter than.

$\Delta V \sim 0.03$ mag $\mathrm{yr}^{-1}$, respectively, thus a whole $1 \mathrm{mag}$ for HM Sge and $0.6 \mathrm{mag}$ for V1016 Cyg in 20 years.

On the contrary, K 3-9 has been very stable over the last half century: Table 2 data, Palomar DSS-1 and DSS-2 blue plates $(B=18.25$ on JD $=2434895$, and $B=18.30$ on JD $=2445936)$ as well as mean values for 1999 from Henden \& Munari (2000) all cluster tightly around $B=18.3$, as shown in Fig. 4. The absence of any descending trend and the $\Delta B=1.1$ bright phase in 1959 are more reminiscent of classical symbiotic stars with moderate active phases than of symbiotic Miras in outburst. K 3-9 bears differences with symbiotic Miras in outburst also in the spectra: observations included in the multi-epoch spectrophotometric atlas of symbiotic stars by Munari \& Zwitter (2001) indicate a moderate ionization of a radiation-bounded circumstellar environment, quite different from the situation in HM Sge, V1016 Cyg and other symbiotic Miras in outburst characterized by firework displays of emission line spectra. 


\subsection{V407 Cyg}

Discovered as Nova Cyg 1936, V407 Cyg attracted interest when (a) Meinunger (1966) reported that the main cause of the object's large variability was the presence of a Mira with a 745 day pulsation period (too long for normal field Miras), and (b) unpublished spectra by different authors described a symbiotic star spectrum appearing from time to time (e.g. Duerbeck 1987). Munari et al. (1990) investigated V407 Cyg lightcurve up to late 80ies, confirming the presence of a Mira and discovering a large modulation of the system mean brightness by $\Delta B \sim 3$ mag. A possible 43 year periodicity was derived for the latter and interpreted as the system orbital period in the framework of the Whitelock (1987) model of variable dust obscuration. However, the lightcurve analyzed by Munari et al. spanned 51 years or just one possible orbital period. Thus the search for blue plates obtained earlier than 1935 with astrographs able to reach $B=16$ would be essential to investigate the secular behaviour of mean brightness and then to confirm the possible 43 year periodicity.

Pulsation periods as long as that of V407 Cyg almost invariably pertain to the central star of $\mathrm{OH} / \mathrm{IR}$ sources, engulfed by extremely thick dust cocoons that make these objects invisible at blue wavelengths. If the presence of the accreting and outbursting white dwarf companion inhibits the formation of all but an optically thin circumstellar dust shell (Kolotilov et al. 1998), then V407 Cyg would allow an unobstructed view of the central star of an $\mathrm{OH} / \mathrm{IR}$ source. The dust could partially form and survive only in the shadow cone produced by the Mira itself, which blocks the destroying action of the white dwarf hard radiation field. When, during the orbital motion, the Mira passes at the inferior conjunction and the whole system is seen through the dust cone, the optical pulsation curve is shifted toward fainter magnitudes. In such a framework, the minimum of mean $B$ brightness in the early 70 s would correspond to passage of the Mira at the inferior conjunction.

A new outburst was discovered in 1994 (Munari et al. 1994), 58 years after the discovery event in 1936. At that time the Mira's spectrum appeared severely veiled by a hot continuum in the blue and strong lines of Hydrogen, HeI, HeII, [OIII] erupted in emission, settling the classification of V407 Cyg as a symbiotic star.

The results of our searching the Asiago plate archive for V407 Cyg are presented in Fig. 5. Plates spanning a 40 year interval have been located. The $B$ band data are plotted in a zoomed view in Fig. 6 (top panel) and a $\Delta \mathrm{mag}=8$ amplitude sinusoid following the original Meinunger (1966) ephemeris

$\operatorname{Max}(B)=2429710+745 \times E$

is overplotted for reference. A 43 year, $\Delta$ mag $=2.1$ amplitude modulation is superimposed to represent the dust obscuration phase that peaked in 1973. The correspondence between observed points and the sinusoidal approximation is not impressive, for at least two reasons: (a) irregular variability by the accreting white dwarf may contribute significantly to the overall system $B$ band brightness (apart from the 1936 and 1994 outbursts), and (b) the Mira in V407 Cyg does not follow regular pulsation cycles characterized by similar and in-phase lightcurves (the VSNET and VSOLJ amateur estimate databanks clearly show quite perturbed lightcurves over the last fifteen years).

The complexity of the Mira pulsations in V407 Cyg is evident when its behaviour at different wavelengths is compared (Fig. 6). The lightcurve in the $B$ band is only moderately periodic, while the periodicity looks instead almost perfect in our $R$ and $I$ data. However, the shape and period of the pulsation lightcurve change with the wavelengths. The $R, I, J$ and $K$ lightcurves in Fig. 6 are computed according to the following ephemerids:

$$
\begin{aligned}
& \operatorname{Max}(R)=2429710+745 \times E \\
& \operatorname{Max}(I)=2446050+747.5 \times E \\
& \operatorname{Max}(J)=2446100+752 \times E \\
& \operatorname{Max}(K)=2446150+755.6 \times E
\end{aligned}
$$

where the original $B$-band ephemeris of Meinunger (1966) was found to be the best performing one for the $R$ data and the Kolotilov et al. (1998) ephemeris the best for the $J$ data. They are however not satisfactory for $I$ and $K$ data, for which we determined new periodicities with converging results by different techniques (Fourier and phase dispersion minimization). The apparent increase of the pulsation period (by $1.4 \%$ ) and shift of phase of maximum (by $13 \%$ ) going from $R$ to $K$ wavelengths seems a robust one, as indicated by the poor results that are obtained if the $R$ data are phase plotted against Eq. (8) or the $K$ data against Eq. (5).

The differences highlighted by Eqs. (5)-(8), whose validity could vanish outside the time-span covered by the data used to derive them, might be related to the highly unusual evolutionary state of the Mira in V407 Cyg, resembling the central star of $\mathrm{OH} / \mathrm{IR}$ sources. Beating among phenomena characterized by different periodicities and effective temperatures could induce the above shifts in phase and period. Optical depth effects in the Mira's atmosphere and circumstellar dust envelope could add to the overall picture: viewing the system at different wavelengths means different transparencies of the circumstellar dust and different effective photospheric levels in the stellar atmosphere.

\subsection{V335 Vul, FG Ser, Draco C-1}

The remaining program stars are only briefly commented upon.

V335 Vul was discovered by Dahlmark (1993) to contain a carbon Mira of 342 day pulsation period. On the basis of marked spectral changes and the emission line 

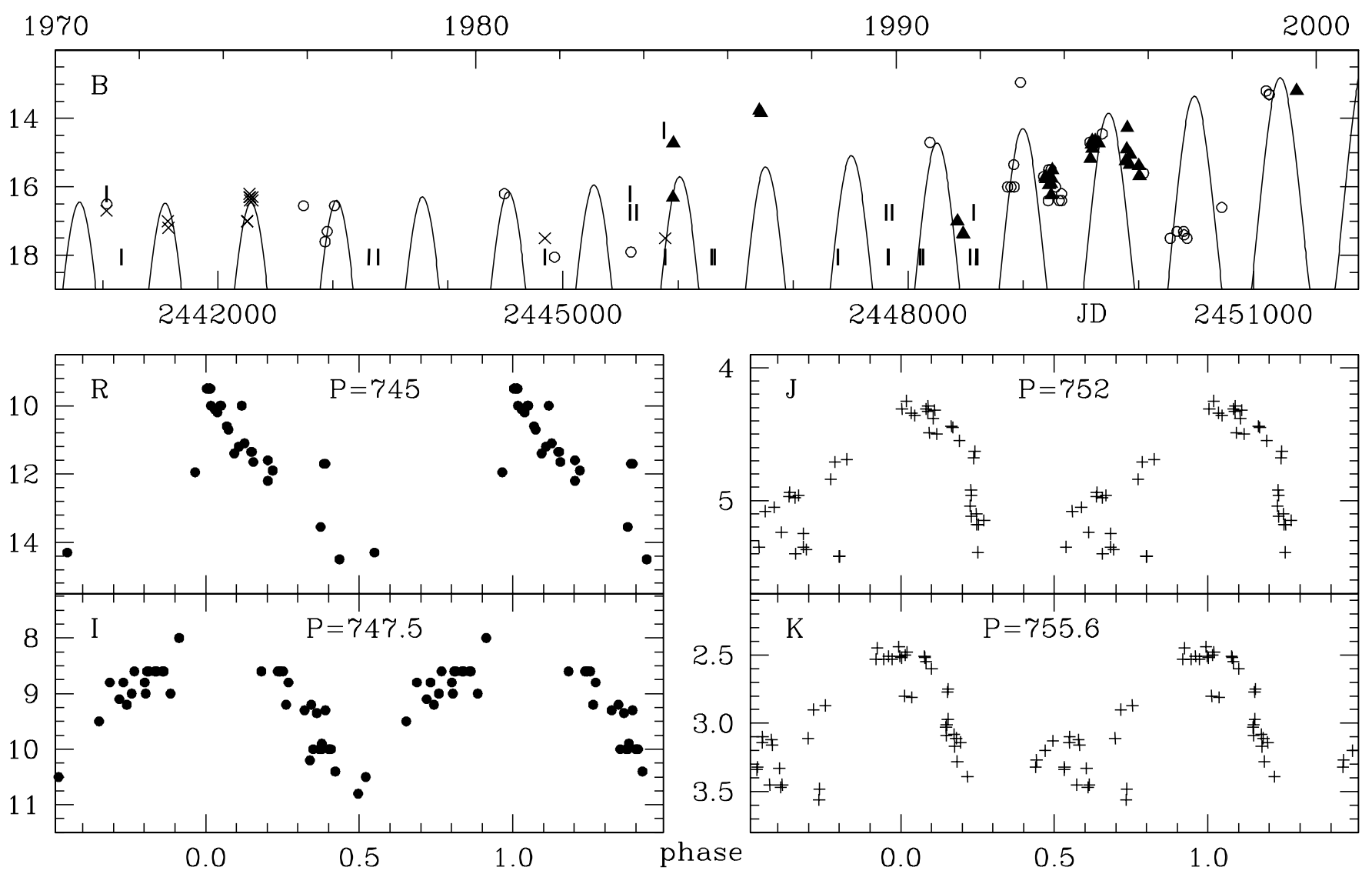

Fig. 6. Upper panel: B lightcurve of V407 Cyg with overplotted a sinusoid phased according to Eq. (4) and modulated in mean brightness with a 43 year periodicity. Lower panels: phase plot of $R$ and $I$ data from Table 2 and $J$ and $K$ data from Kolotilov et al. (1998) according to Eqs. (5)-(8).

spectrum, Munari et al. (1999) suggested a possible symbiotic nature for this object. Our $B$ and $V$ data in Table 2 reveal a variability of significant amplitude, but the resulting lightcurve is disappointingly restricted to a narrow range. This is caused by the short time span covered by the plates (a couple of years), the long pulsation period (close to one year), and the seasonal visibility.

The photometric and outburst history of the eclipsing symbiotic star FG Ser has already been reviewed in detail by Munari et al. $(1992,1995)$ and Kurochkin (1993), and it will not be further commented upon here. Part of the plates here used to derive the magnitude of FG Ser were already investigated by Munari et al., but they are measured again here both to place them on the more accurate Henden \& Munari (2000) photometric sequence and to link them with the more recent data reported in Table 2.

Similarly, Draco C-1 plate material prior to 1991 has already been considered by Munari (1991). It has been estimated again here against the better Henden \& Munari (2000) comparison sequence, in order to place it on the same scale as the data collected after 1991 and included in Table 2. The star is very close to the limiting magnitude on all plates, and this affects both star detection and brightness estimate. A significant $\Delta I=0.7 \mathrm{mag}$ variability is however evident, apparently not much correlated with $B$ behavior. Apart from a $\Delta B=1.3$ mag bright phase in 1988, Draco C-1 remained quiescent around $B=18.4$ over 1987-1996, close to the mean $B=18.6$ measured in 1999 by Henden \& Munari (2000) and the $B=18.43$ measured in 1981 by Aaronson et al. (1982).

Acknowledgements. RJS wish to thank the hospitality of the Asiago observatory and the Primorsko-Goranska County for financial support.

\section{References}

Allen, D. A. 1974, MNRAS, 168, 1

Allen, D. A. 1984, PASAu, 5, 369

Aaronson, M., Liebert, J., \& Stocke, J. 1982, ApJ, 254, 507

Arkhipova, V. P., \& Noskova, R. I. 1988, SvA Lett., 14, 188

Arkhipova, V. P., Ikonnikova, N. P., \& Noskova, R. I. 1995, PAZh, 21, 379

Carrasco, L., Costero, R., \& Serrano, P. G. 1983, in Planetary Nebulae, ed. D. R. Flower, Proc. of IAU Symp., 103, 548

Dahlmark, L. 1993, IBVS, 3855

Duerbeck, H. W. 1987, A Reference Catalogue and Atlas of Galactic Novae (Reidel, Dordrecht)

Henden, A. A., \& Munari, U. 2000, A\&AS, 143, 343

Henden, A. A., \& Munari, U. 2001, A\&A, 372, 145

Henden, A. A., \& Munari, U. 2002, A\&A, submitted

Henize, K. G. 1967, ApJS, 14, 125 
Ivison, R. J., \& Seaquist, E. R. 1995, MNRAS, 272, 878

Kenyon, 1986, The Symbiotic Stars (Cambridge University Press)

Kolotilov, E. A., Munari, U., Popova, A. A., et al. 1998, Astron. Lett., 24, 451

Kurochkin, N. E. 1993, Astron. Astrophys. Trans., 3, 295

Margoni, R., \& Stagni, R. 1984, A\&AS, 56, 87

Meinunger, L. 1966, Mitt. Veranderl. Sterne, 3, 111

Mikolajewska, J. 1996, Physical Processes in Symbiotic Binaries and Related Objects (editor, Polish Academy of Sciences, Warsaw)

Munari, U. 1991, A\&A, 251, 103

Munari, U., \& Buson, L. M. 1991, A\&A, 249, 141

Munari, U., \& Buson, L. M. 1992, A\&A, 255, 158

Munari, U., \& Zwitter, T. 2002, A\&A, 383, 188

Munari, U., Bragaglia, A., Guarnieri, M. D., et al. 1994, IAUC, 6049
Munari, U., Jurdana-Šepić, R., \& Moro, D. 2001, A\&A, 370, 503 (Paper I)

Munari, U., Margoni, R., \& Stagni, R. 1990, MNRAS, 242, 653

Munari, U., Tomov, T., \& Rejkuba, M. 1999, Inf. Bull. Var. Stars, 4668

Munari, U., Rejkuba, M., Mattei, J., et al. 1997, A\&A, 323, 113

Munari, U., Yudin, B. F., Taranova, O. G., et al. 1992a, A\&AS, 93, 383

Munari, U., Yudin, B. F., Kolotilov, E., \& Gilmore, A. 1995, AJ, 109, 1740

Munari, U., Whitelock, P. A., Gilmore, A. C., et al. 1992b, AJ, 104,262

Passuello, R., Saccavino, S., \& Munari, U. 1994, IAUC, 6065

Whitelock, P. A. 1987, PASP, 99, 573 